\title{
28 Research Square \\ Concealing messages at the atomic-thin level by reaching the limit of writing
}

Chao Hou

Peking University

Qirong Yang

Peking University

Jianxin Guan

Peking University https://orcid.org/0000-0002-8984-7384

Jingwen Deng

Peking University

Zihan Xu

Shenzhen Six Carbon Tech Co. Ltd

Zhihao Yu

Peking University https://orcid.org/0000-0002-6538-5007

Junrong Zheng ( $\nabla$ junrong@pku.edu.cn )

Peking University

\section{Article}

Keywords: Steganography, Single Molecular Layer Ink, P-type Doping, Photoluminescence, High-density Storage Media

Posted Date: December 8th, 2020

DOI: https://doi.org/10.21203/rs.3.rs-111143/v1

License: (1) This work is licensed under a Creative Commons Attribution 4.0 International License. Read Full License

Version of Record: A version of this preprint was published at Advanced Materials Technologies on October 27th, 2021. See the published version at https://doi.org/10.1002/admt.202101089. 


\section{Abstract}

The evolution of writing system reflects the development of civilization. Using single molecular layer "ink" to record invisible messages on an atomic-layered paper represents the ultimate thinness of classical writing system and steganography. A stable and convenient atomic-thin information steganography under ambient conditions is realized by using a monolayer of water as ink, an atomic layer of MoS2 as paper, and a laser as pen. Physically adsorbed monolayered water molecules produce p-type doping to the substrate MoS2 monolayer, leading to the enhancement of its photoluminescence (PL). Information is written on a monolayer of MoS2 by using laser to remove surface water with designed patterns and is read out by PL imaging. The information is transparent to visible light and can retain for many days until erased by a humidification treatment. The method reaches the thinnest limit of writing and achieves repeatable data storage on an atomic layer under ambient conditions, providing a promising approach to steganography and towards high-density storage media at the atomic-thin level.

\section{Main Text}

From prehistoric symbolic murals carved on caves by stone, to the confidential information written on letter paper by invisible ink, the continuous improvement of writing system has witnessed human progresses. A special type of writing, steganography, which is usually taken as a top secret, is evolving with time. The formulas of secret ink used in the First World War were ordered to remain hidden from the public in $2002^{1}$, even though these secret writing methods merely stay at the primary level of chromogenic reaction. At the turn of this century, it was reported that the steganographic technique could be advanced to the molecular level by concealing secret messages in DNA-based microdot ${ }^{2,3}$ and macromolecules ${ }^{4}$. By thinning down paper and ink to the atomic scale, one may reach the limit of writing system and take steganography a step further, even enabling the storage of data in an atomic-thin memory. The path to store information at the atomic limit can be carried out by scanning probe microscopes (SPM), e.g. scanning tunneling microscope (STM) through directly manipulating lattice locations ${ }^{5,6}$, charge states ${ }^{7,8}$ or magnetization states ${ }^{9-11}$, or atomic force microscope (AFM) methods like dip-pen nanolithography (DPN) ${ }^{12-14}$ that can deliver a wide range of substances onto various substrates $^{13,15,16}$. The SPM methods require low temperature, clean surface, ultrahigh vacuum environment and/or complicated operations ${ }^{17,18}$, and their information readout typically relies on delicate SPM or electronic microscopes. These are undesirable features for steganography of which users are usually under intense pressure with limited conditions and have limited time to work.

Herein, using a monolayer of water as ink, an atomic layer of $\mathrm{MoS}_{2}$ as paper, and a laser as pen, concealing information at the atomic-thin level is achieved under ambient conditions in a rewritable manner. The optical image of an n-doped $\mathrm{MoS}_{2}$ monolayer is displayed in Fig. 1a. Its PL is enhanced upon water absorption, and the desorption of water by laser irradiation suppresses the PL enhancement, as shown in Fig. 1b. Raman spectroscopic measurements suggest that the laser irradiation does little damage to the $\mathrm{MoS}_{2}$ monolayer. As displayed in Fig. 1c, absorbing $\mathrm{H}_{2} \mathrm{O}$ molecules blueshifts and 
intensifies the out-of-plane vibrational mode $\mathrm{A}_{1}{ }^{\prime}$ of $\mathrm{MoS}_{2}$, whereas the in-plane vibrational mode $\mathrm{E}^{\prime}$ stays unchanged (blue line). After the laser irradiation treatment, the Raman spectrum (red line) changes back to be almost identical to the pristine one (black line), suggesting that there is little structural change within the monolayer of $\mathrm{MoS}_{2}$. To investigate the mechanism of PL enhancement by absorbing $\mathrm{H}_{2} \mathrm{O}$ molecules, a field-effect transistor (FET) device is fabricated to explore the variations in carrier concentration of $\mathrm{MoS}_{2}$. Fig. 1d\&e display the optical images of the FET device before and after adsorbing $\mathrm{H}_{2} \mathrm{O}$ molecules, respectively. Their appearances look the same, but their electronic properties vary significantly. The source-drain current $\left(\mathrm{I}_{\mathrm{SD}}\right)$ versus back gate voltage $\left(\mathrm{V}_{\mathrm{G}}\right)$ curves in Fig. $1 \mathrm{f}$ indicate that the pristine device (black curve) shows an n-type characteristic. After absorbing $\mathrm{H}_{2} \mathrm{O}$ molecules on the surface, the electron concentration drops sharply (blue curve), suggesting that the physically adsorbed $\mathrm{H}_{2} \mathrm{O}$ molecules have a p-type doping to $\mathrm{MoS}_{2}$. A p-type doping favors the formation and recombination of neutral excitons over negative trions. According to literatures ${ }^{19-21}$, neutral excitons have a faster radiative recombination rate than the negative trions and thus have a higher $P L$ efficiency, leading to the $P L$ enhancement. Once the absorbed $\mathrm{H}_{2} \mathrm{O}$ molecules are erased by annealing in vacuum $\left(20{ }^{\circ} \mathrm{C}\right.$ for $3 \mathrm{~h}$ ), the n-type characteristic (green curve) is restored, and in fact is even slightly enhanced compared to the pristine sample, because fewer water molecules are on the annealed sample.

The laser irradiation removing the surface $\mathrm{H}_{2} \mathrm{O}$ molecules is further verified with AFM mapping. A square region by laser irradiation on the $\mathrm{MoS}_{2}$ sample (the blue rectangle area denoted in Fig. 1a) is not distinguishable by optical microscope, whereas it is clearly visible under AFM mapping (Fig. 1g). The line profile in Fig. $1 \mathrm{~h}$ indicates that the thickness of the monolayer $\mathrm{MoS}_{2}$ before laser treatment is $1.43 \mathrm{~nm}$, which is higher than the theoretical value of monolayered $\mathrm{MoS}_{2}$ owing to the adsorbed water. The average thickness of the laser-treated square region (Fig. 1i) is measured to be $0.75 \mathrm{~nm}$, consistent with the reported monolayer thickness of $\mathrm{MoS}_{2}{ }^{22}$. The thickness reduction caused by laser treatment is about $0.68 \mathrm{~nm}$, which agrees well with the thickness of a single layer of water molecules $(\sim 0.4 \mathrm{~nm})$ plus the adsorption equilibrium distance $(\sim 0.2 \mathrm{~nm})^{23}$. The measured thickness of $\mathrm{MoS}_{2}$ monolayer with absorbed water is consistent with previous theoretical studies ${ }^{24,25}$.

By removing surface water in designed patterns with lasers and the re-addition of water by humidification, rewritable data-encoding and readout through simple PL mapping can be achieved. The encoding scheme is illustrated in Fig. 2a. The sample is moisturized before laser writing, and then patterns are written directly onto the $\mathrm{MoS}_{2}$ monolayer with a laser by removing the surface water. $\mathrm{A}$ home-built laser writing system is used to conduct the information-writing process (Supplementary Fig. 1). The laser-written information is read out by PL imaging, and is erased by exposing the sample to a humid environment. Fig. $2 \mathrm{~b}$ displays a Chinese character " $\nabla$ " with the meaning of benevolence that is written onto the $\mathrm{MoS}_{2}$ monolayer. The writing of " $\nabla$ " (Fig. 2c) and the erasing of it with water absorption (Fig. 2d) don't produce observable changes on the sample's optical images. Through PL mapping, the written pattern is clearly visible (Fig. 2 e). Under ambient conditions (humidity $<30 \%$ ), the written pattern can last for at least one week. The pattern is successfully erased after the sample is placed in a humid 
environment (relative humidity $~ 85 \%$ for about 10 minutes) (Fig. 2f). The PL spectra of the sample before any treatments (black curve), after laser writing (red curve), and after pattern-erasing by adsorbing $\mathrm{H}_{2} \mathrm{O}$ molecules (blue curve) are displayed in Fig. $2 \mathrm{~g}$. They are very similar to those in Fig. $1 \mathrm{~b}$, of which the PL intensity is dependent on the surface water content as discussed above. During writing, the laser power density is carefully controlled at about $3.0 \times 10^{5} \mathrm{w} / \mathrm{cm}^{2}$ to ensure that the thermal energy generated by $\mathrm{MoS}_{2}$ photo-absorption is sufficient for the removal of water but below the $\mathrm{MoS}_{2}$ damage threshold. Overwriting with too much laser power can cause permanent damages to the $\mathrm{MoS}_{2}$ monolayer and the written pattern cannot be erased by adsorbing $\mathrm{H}_{2} \mathrm{O}$ molecules (Supplementary Fig. 2).

The $\mathrm{MoS}_{2}$ monolayer is rewritable for many times. Fig. 3 displays the PL images of " $\nabla$ " written in the solid-filled form (upper panels) and erased (bottom panels) at the $1^{\text {st }}, 10^{\text {th }}, 20^{\text {th }}, 50^{\text {th }}$ cycle. The upper panels are taken after each writing. " $\square$ " is still visible after 50 writing/erasing cycles. The PL images in the bottom panels are taken after each humidification treatment, showing that " $\nabla$ " is completely removed by the erasing process. To test how long the written pattern can last, we keep the sample in a container at $30 \%$ relative humidity $(\mathrm{RH})$ for a week right after the $20^{\text {th }}$ writing. Water in the air has not been able to erase the written pattern for such a long period of time, and " $\nabla$ " is still clearly distinguishable, as shown in the upper $20^{\text {th }}$ panel in Fig. 3. The corresponding optical images of the sample after each writing/erasing cycle also show no noticeable changes on the $\mathrm{MoS}_{2}$ monolayer during the processes (Supplementary Fig. $3)$.

Not only can a simple word or symbol be written with the method, but also a sentence and even a painting. The phrase "Nothing is Impossible" (Fig. 4c) and the famous painting Mona Lisa (Fig. 4f) are successfully written on continuous $\mathrm{MoS}_{2}$ monolayered films. The line drawing of Mona Lisa (Fig. 4f) is probably the thinnest portrait by far, since the "paper" is a monolayer of $\mathrm{MoS}_{2}$ and the "pigment" is one layer of $\mathrm{H}_{2} \mathrm{O}$ molecules.

The writing/erasing process is reasonably fast, dependent on the complexity of the writen pattern. A more complexed pattern takes a longer time. With the same condition, writing " $\nabla$ " and " $\pi$ " of a similar size takes about $60 \mathrm{~s}$ and $15 \mathrm{~s}$, respectively. It takes shorter than 10 mins to obtain their PL images by mapping. The erasing can be extremely fast. In a destructive way, if the sample is dipped into water or blown by breath, the pattern disappears immediately, but, in most of our tries the $\mathrm{MoS}_{2}$ monolayer is broken after the erasing process. In a nondestructive manner, the sample is placed in an environment with a $\mathrm{RH}$ of $85 \%$, the written pattern disappears within 10 minutes. In total, it takes minutes for the entire writing/erasing/readout process. The time can be significantly shortened by optimizing the operation conditions and tools, e.g. using 2D PL imaging rather than point mapping used in this work can cut the readout time from minutes to seconds, and using a pulsed laser that can deliver enough heat to desorb water molecules before the thermal energy diffuses out of the focus spot would increase both the writing speed and the spatial resolution. 
In summary, atomic-thin erasable and rewritable information recorded on a $\mathrm{MoS}_{2}$ monolayer under ambient conditions is achieved by modulating and imaging the PL of a MoS 2 monolayer with surface water reversible absorption/desorption. The messages encoded in this way can be at least under triple protections: information is encrypted, the writing appearance is invisible, and the designated $\mathrm{MoS}_{2}$ triangle is camouflaged among a jumble of $\mathrm{MoS}_{2}$ triangles; and they can be easily and immediately erased by breath or even saliva. The miniaturization of the equipment needed for the writing/readout processes is feasible and their operations can be foolproof. These are desirable and promising features for many applications, e.g. steganography, anti-counterfeiting, sensors, and transient information storage.

\section{References}

1. United States District Court for the District of Columbia, The James Madison Project v. National Archives \& Records Administration, CA98-2737, 2002.3.5.

2. Clelland, C., Risca, V. \& Bancroft, C. Hiding messages in DNA microdots. Nature 399, 533-534 (1999)

3. Goldman, N. et al. Towards practical, high-capacity, low-maintenance information storage in synthesized DNA. Nature 494, 77-80 (2013).

4. Cavallini, M. et al. Information Storage Using Supramolecular Surface Patterns. Science 299, 531531 (2003).

5. Bennewitz, R. et al. Atomic scale memory at a silicon surface. Nanotechnology 13, 499-502 (2002).

6. Kalff, F. E. et al. A kilobyte rewritable atomic memory. Nature Nanotechnology 11, 926-929 (2016).

7. Garcia, V. \& Bibes, M. Ferroelectric tunnel junctions for information storage and processing. Nature Communications 5, 1-12 (2014).

8. Repp, J., Meyer, G., Olsson, F. E. \& Persson, M. Controlling the Charge State of Individual Gold Adatoms. Science 305, 493-495 (2004).

9. Natterer, F. D. et al. Reading and writing single-atom magnets. Nature 543, 226-228 (2017).

10. Loth, S., Baumann, S., Lutz, C. P., Eigler, D. M. \& Heinrich, A. J. Bistability in Atomic-Scale Antiferromagnets. Science 335, 196-199 (2012).

11. Kiraly, B. et al. An orbitally derived single-atom magnetic memory. Nature Communications 9, 1-8 (2018).

12. Piner, R. D., Zhu, J., Xu, F., Hong, S. \& Mirkin, C. A. 'Dip-Pen' Nanolithography. Science 283, 661-663 (1999).

13. Ginger, D. S., Zhang, H. \& Mirkin, C. A. The Evolution of Dip-Pen Nanolithography. Angewandte Chemie International Edition 43, 30-45 (2004).

14. Salaita, K., Wang, Y. \& Mirkin, C. A. Applications of dip-pen nanolithography. Nature Nanotechnology 2, 145-155 (2007).

15. Ivanisevic, A. \& Mirkin, C. A. “Dip-Pen” Nanolithography on Semiconductor Surfaces. J. Am. Chem. Soc. 123, 7887-7889 (2001). 
16. Liu, G., Hirtz, M., Fuchs, H. \& Zheng, Z. Development of Dip-Pen Nanolithography (DPN) and Its Derivatives. Small 15, 1900564 (2019).

17. Eigler, D. M. \& Schweizer, E. K. Positioning single atoms with a scanning tunnelling microscope. Nature 344, 524-526 (1990).

18. Stroscio, J. A. \& Eigler, D. M. Atomic and Molecular Manipulation with the Scanning Tunneling Microscope. Science 254, 1319-1326 (1991).

19. Mouri, S., Miyauchi, Y. \& Matsuda, K. Tunable Photoluminescence of Monolayer MoS2 via Chemical Doping. Nano Lett. 13, 5944-5948 (2013).

20. Lien, D.-H. et al. Electrical suppression of all nonradiative recombination pathways in monolayer semiconductors. Science 364, 468-471 (2019).

21. Nan, H. et al. Strong Photoluminescence Enhancement of MoS2 through Defect Engineering and Oxygen Bonding. ACS Nano 8, 5738-5745 (2014).

22. Tan, C. et al. Recent Advances in Ultrathin Two-Dimensional Nanomaterials. Chem. Rev. 117, 62256331 (2017).

23. Yue, Q., Shao, Z., Chang, S. \& Li, J. Adsorption of gas molecules on monolayer MoS2 and effect of applied electric field. Nanoscale Res Lett 8, 425 (2013).

24. Schumacher, A., Scandella, L., Kruse, N. \& Prins, R. Single-layer MoS2 on mica: studies by means of scanning force microscopy. Surface Science 289, L595-L598 (1993).

25. Mieda, E. et al. Nanoprobe characterization of MoS2 nanosheets fabricated by Li-intercalation. Japanese Journal of Applied Physics 54, 08 LB07 (2015).

\section{Methods}

Growth of monolayer $\mathrm{MoS}_{2}$. Monolayer $\mathrm{MoS}_{2}$ was grown on clean $285 \mathrm{~nm}$ thick $\mathrm{SiO}_{2} / \mathrm{Si}$ wafers in a twotemperature-zone furnace via CVD method. $\mathrm{MoO}_{3}$ powder (Alfa Aesar, $99.999 \%, 0.01 \mathrm{~g}$ ) and sulfur powder (Alfa Aesar, 99.999\%, $0.3 \mathrm{~g}$ ) were used as solid sources. The crucible containing $\mathrm{MoO}_{3}$ power was placed $30 \mathrm{~cm}$ downstream of the sulfur boat. The $\mathrm{SiO}_{2} / \mathrm{Si}$ substrate was positioned facing down at the location of $3 \mathrm{~cm}$ downstream from the $\mathrm{MoO}_{3}$ boat. During growth, $300 \mathrm{sccm}$ Ar gas was used as the carrier gas. After purging Argas for $20 \mathrm{~min}$, the temperature for $\mathrm{MoO}_{3}$ and sulfur powders was raised to $650{ }^{\circ} \mathrm{C}$ and $180^{\circ} \mathrm{C}$, respectively. Then the monolayer $\mathrm{MoS}_{2}$ was obtained after $\sim 7$ mins of growth.

Laser writing and erasing. We used a home-built laser system to process the laser writing operation. The optical setup is illustrated in Supplementary Fig. 1, consisting of a microscopic system and a galvanometric scanning system. A continuous-wave solid-state laser diode ( $532 \mathrm{~nm}$ ) was equipped as the laser writing source. We used a $60 \times$ objective lens to focus the laser spot into around $2.0 \mu \mathrm{m}$. The laser writing path according to the chosen pattern was designed by the software Autodesk (Autodesk Fusion 360). All laser writing processes were performed by using $\sim 8 \mathrm{~mW}$ laser power at room temperature in ambient air. In particular, the laser spot should be accurately focused on the surface of the 
monolayer $\mathrm{MoS}_{2}$ to guarantee that the message can be effectively read and erased. The messages can be maintained at $30 \% \mathrm{RH}$ at least one week. The erasing treatment was carried out by another humidification treatment.

PL/Raman and AFM measurements. In this work, we used a confocal Raman microscopic system (HORIBA, XploRA Plus) equipped with a $532 \mathrm{~nm}$ laser and a 100x objective lens to obtain PL mappings. The focused laser spot size on the sample was about $0.6 \mu \mathrm{m} .600 \mathrm{gr} / \mathrm{mm}$ gratings, $100 \mu \mathrm{m}$ slit and 300 $\mu \mathrm{m}$ hole were adapted. The mapping step size is $0.3 \mu \mathrm{m}$ with swift-on mode, and the integration time on each point is $0.04 \mathrm{~s}$. All measurements were performed by using $\sim 0.5 \mathrm{~mW}$ laser power at room temperature in ambient air. For the single point PL/Raman spectra, we used our home-built PL/Raman microspectroscopy with a $532 \mathrm{~nm}$ laser source and 50x long-working-distance objective lens ( $0.7 \mathrm{NA})$ which provides a spot size of $\sim 2.5 \mu \mathrm{m}$. Typical excitation power was $5 \mathrm{~mW}$, and the integration time was $10 \mathrm{~s}$. We used $600 \mathrm{gr} / \mathrm{mm}$ grating and $300 \mu \mathrm{m}$ slit to obtain PL spectra, and used $1800 \mathrm{gr} / \mathrm{mm}$ grating and $50 \mu \mathrm{m}$ slit to acquire Raman spectra. Thickness variations of the samples were conducted with the tapping mode by using a commercial atomic force microscope (AFM, Bruker Dimension Icon with Nanoscope $V$ controller).

Device fabrication and testing. To fabricate the FET device, polymethyl methacrylate (PMMA, Microchem, $495 \mathrm{~K}, \mathrm{A6}$ ) was firstly spin-coated onto the sample as the mask. We adapted Auto CAD to design an area in order to remove the undesired triangle domains within a $200 \times 200 \mu \mathrm{m}$ region around the target $\mathrm{MoS}_{2}$ single domain. This designed area was then exposed by conducting high-resolution electron beam lithography (EBL). Through a precise oxygen plasma etching process, the exposed areas were etched, followed by a subsequent rinse with acetone to remove the top PMMA layer. Next, a second lithography was performed to create the source and drain electrode patterns. Cr/Au (5 nm/ $45 \mathrm{~nm})$ electrodes were thermally evaporated onto the substrate, and then acetone was used to remove the PMMA mask. Finally, the device was annealed in vacuum at $200^{\circ} \mathrm{C}$ for $3 \mathrm{~h}$ to eliminate PMMA residuals and any moisture. The electrical characterizations of the $\mathrm{MoS}_{2}$ FET device were carried out in ambient air on a probe station using a Keithley 4200 SCS as the parameter analyzer.

Data availability The data that support the findings of this study are available from the corresponding author on reasonable request.

\section{Declarations}

Acknowledgements The work is supported by the National Science Foundation of China (NSFC21627805, 21673004, 21804004, and 21821004) and MOST (2017YFA0204702) China. The PL mappings were performed at HORIBA Scientific office (Beijing, China), and the electrical characteristics of FET devices were carried out at Prof. Jian Pei's lab in the college of Chemistry and Molecular Engineering 
in Peking University. The authors thank the help offered by Dr. Yilin Lu, Dr. Teng Tu and Dr. Zefan Yao for the measurements of the mappings and electrical results.

Author contributions C.H. and J.Z. designed experiments. C.H. and Q.Y. conducted experiments. J.G. fabricated the FET devices. J.D. helped develop the PL/Raman microspectroscopy. Z.X. prepared the $\mathrm{MoS}_{2}$ monolayers. Z.Y. designed and built the laser writing system. C.H. and Q.Y. analyzed data. C.H., Q.Y. and J.Z. prepared the manuscript. J.Z. supervised the project. All authors made critical contributions to the work.

Competing interests The authors declare no competing interests.

\section{Additional information}

Correspondence and requests for materials should be addressed to J.Z. (junrong@pku.edu.cn).

\section{Figures}


a
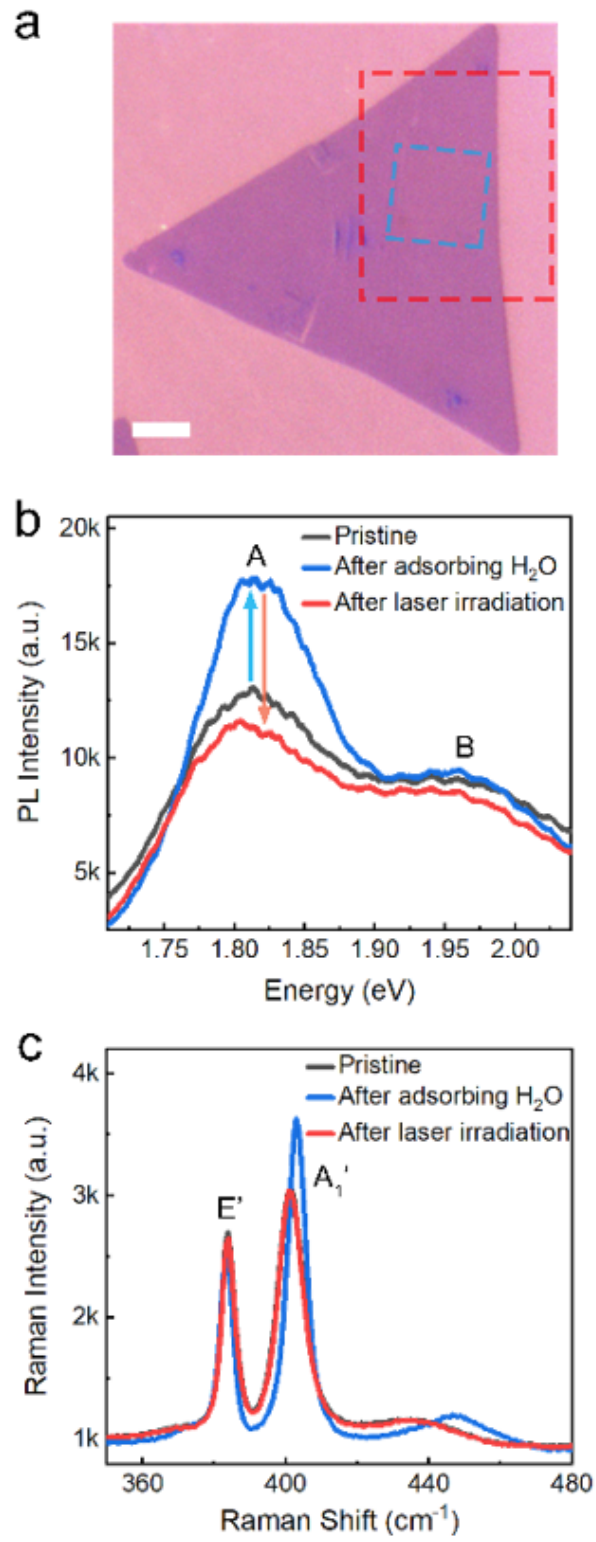

d

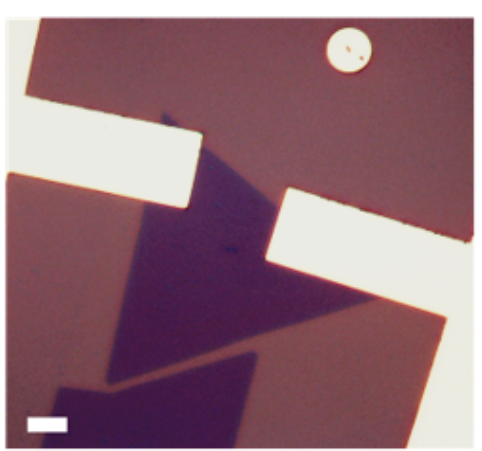

e
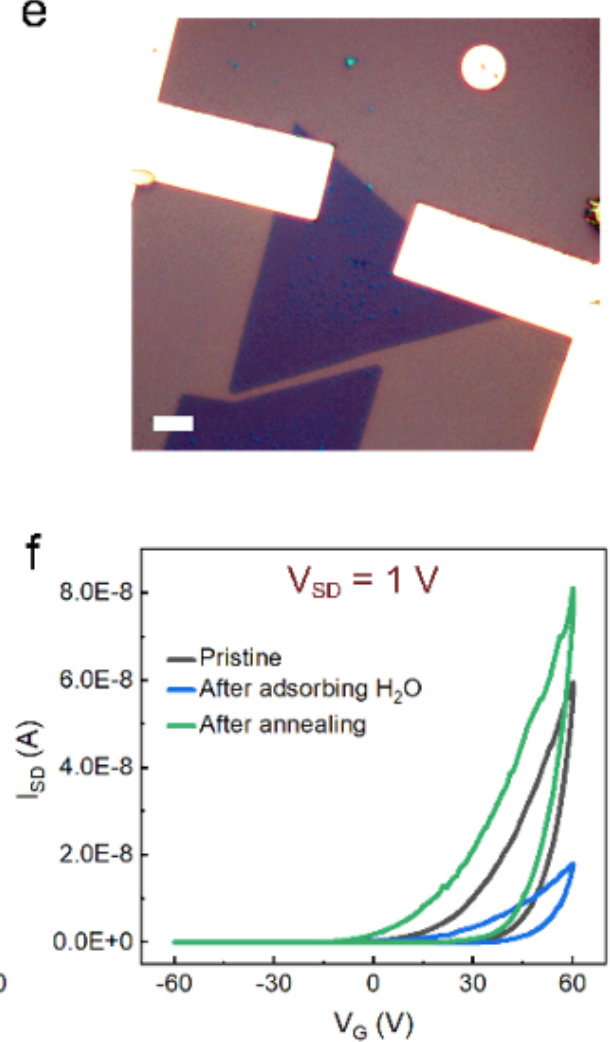

g

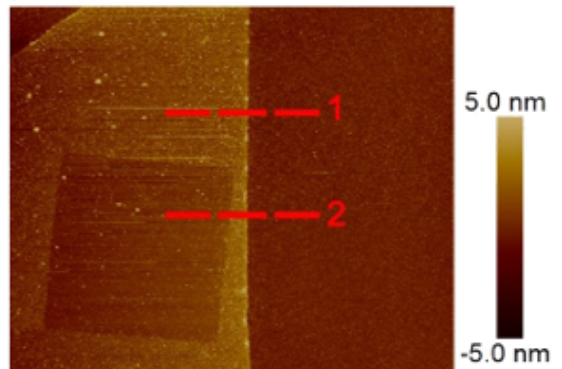

Height Sensor $\overline{5.0 \mu \mathrm{m}}$

$\mathrm{h}$
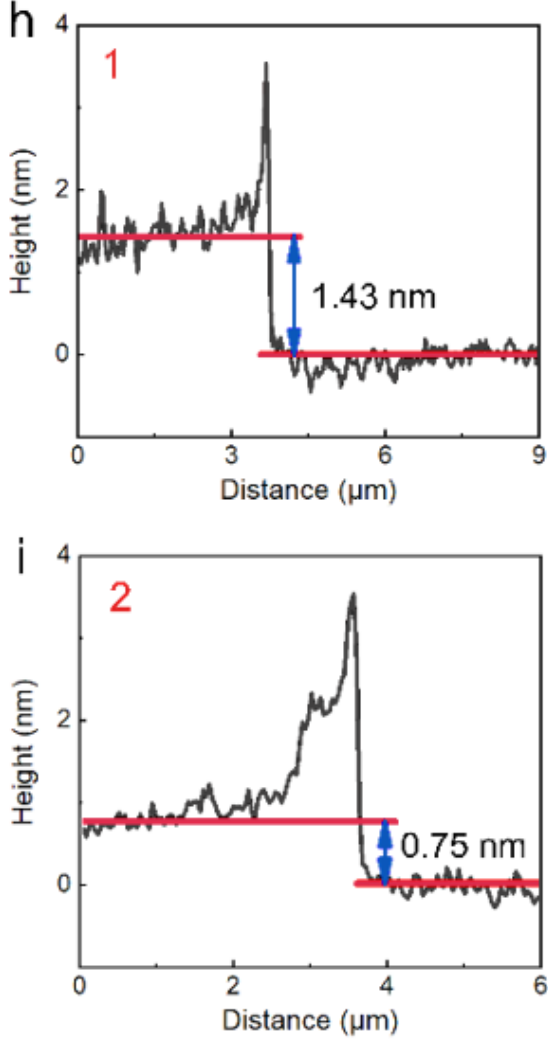

\section{Figure 1}

The PL enhancement and recovery of MoS2 monolayer. a, Optical image of a monolayered MoS2 triangle domain. b\&c, The PL and Raman spectra of the MoS2 monolayer before and after corresponding treatments. d\&e, Optical images of the monolayered MoS2 FET device before and after absorbing $\mathrm{H} 2 \mathrm{O}$ molecules. f, Source-drain current (ISD) versus back gate voltage (VG) curves with a source-drain voltage (VSD) of $1 \mathrm{~V}$ for the FET device before and after corresponding treatments. $\mathrm{g}$, AFM image for the red rectangle area in a, including the laser-treated region denoted with blue rectangle. h\&i, A line profile showing the height along the red line \#1 and \#2 in g. Scale bars are all $5 \mu \mathrm{m}$. 
a

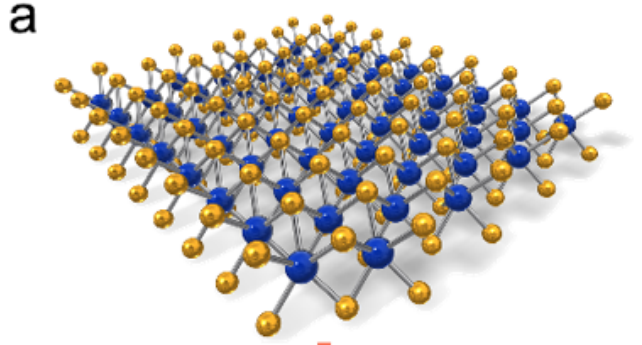

Adsorbing $\mathrm{H}_{2} \mathrm{O}$
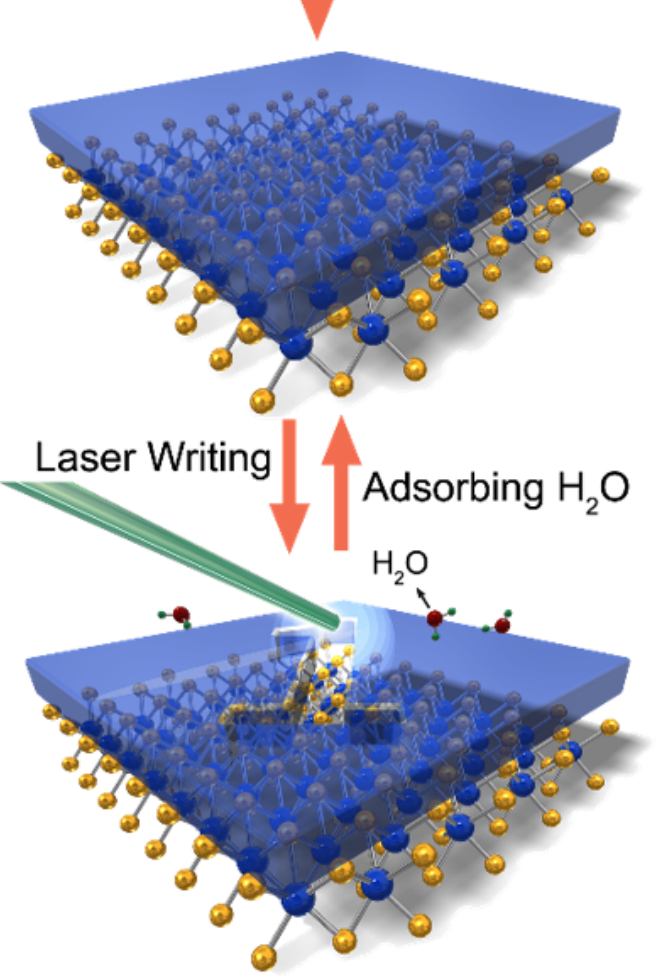

b

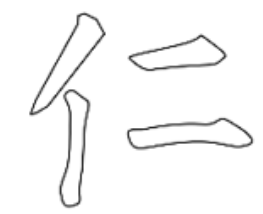

C

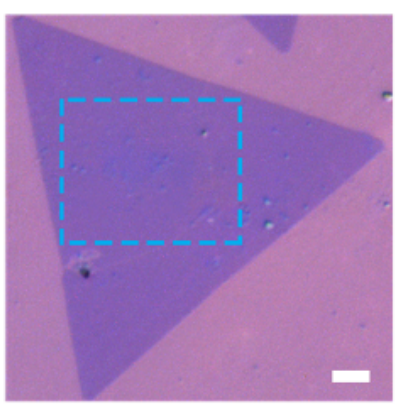

d

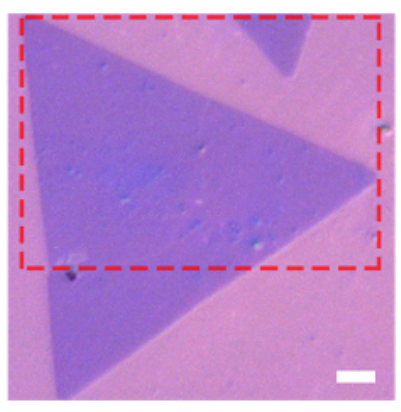

e

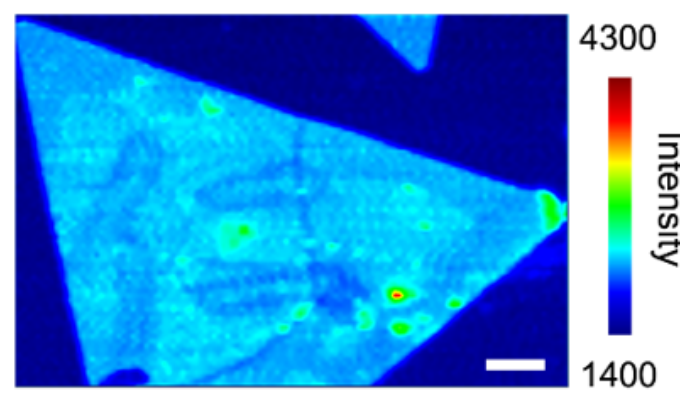

f

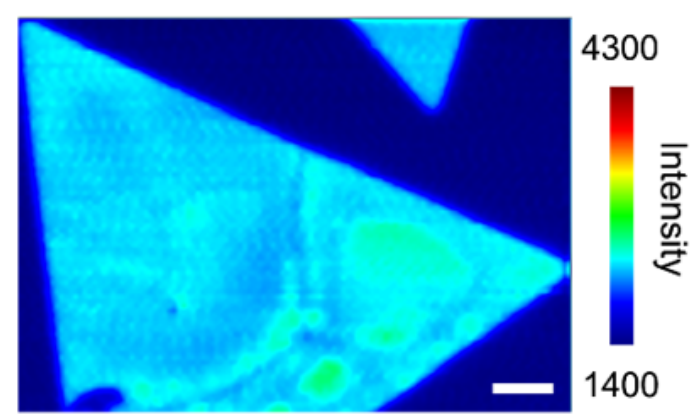

g

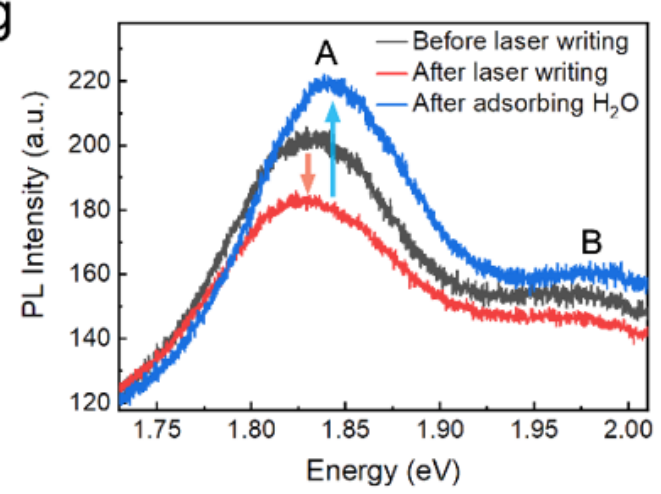

\section{Figure 2}

Laser writing and erasing on MoS2 monolayer. a, A scheme of laser writing and erasing cycle. b, A Chinese character meaning "benevolence" used as a laser irradiation pattern. c, Optical image of a MoS2 monolayer triangle domain after laser writing " $\nabla$ " inside the blue rectangle. $d$, Optical image of $c$ after erasing the pattern by adsorbing $\mathrm{H} 2 \mathrm{O}$ molecules. The red rectangle denotes the area being mapped. e\&f, PL mapping images (at 1.82-2.00 eV) corresponding to $c$ and d, respectively. g, PL spectra of the sample before (black curve) and after laser writing (red curve), and after erasing treatment (blue curve), respectively. Scale bars are all $4 \mu \mathrm{m}$. 


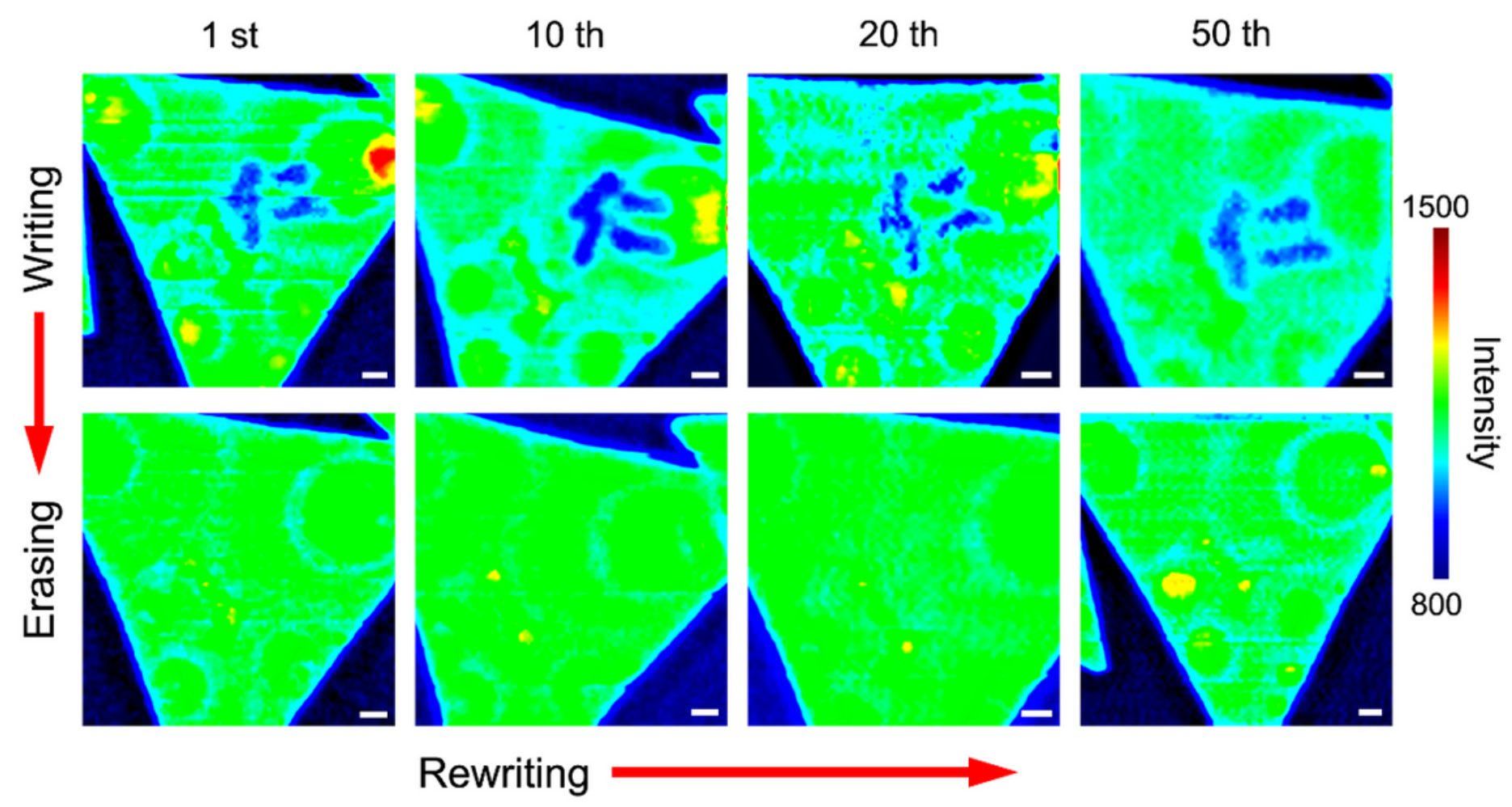

Figure 3

Repeatable laser writing and erasing on MoS2 monolayer. The PL mapping for the 1st, 10th, 20th, 50th laser writing (upper panels) and erasing (bottom panels) " $\square$ " on the same MoS2 monolayer triangle domain. Scale bars are all $2 \mu \mathrm{m}$. 
a

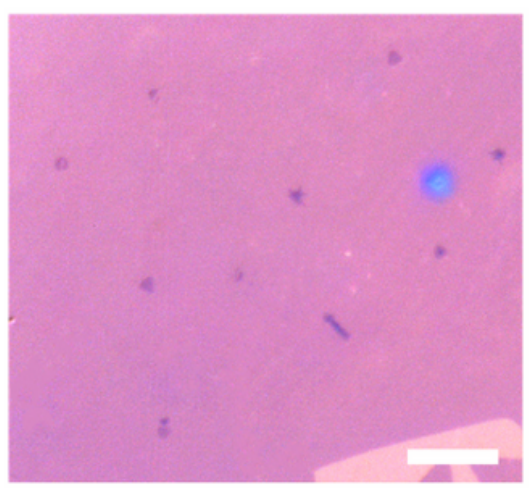

d

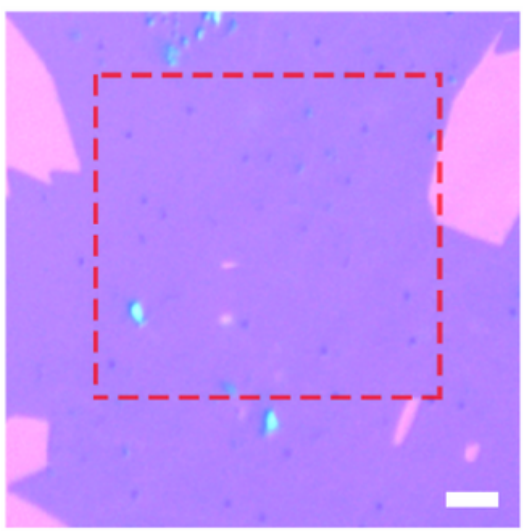

b
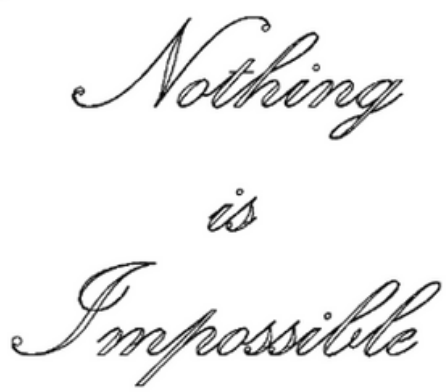

e

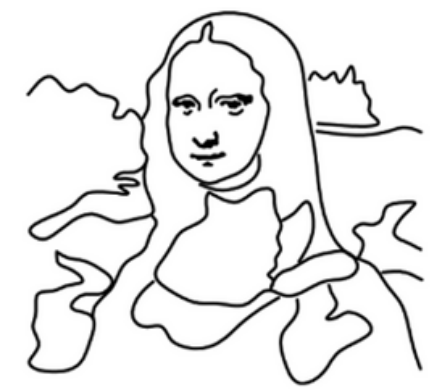

C

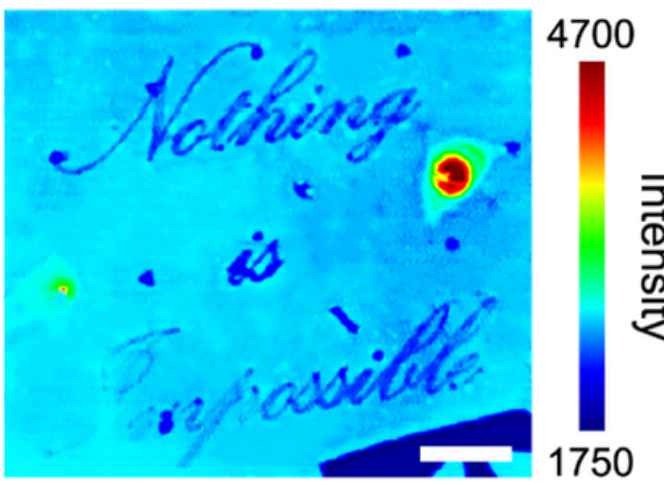

f

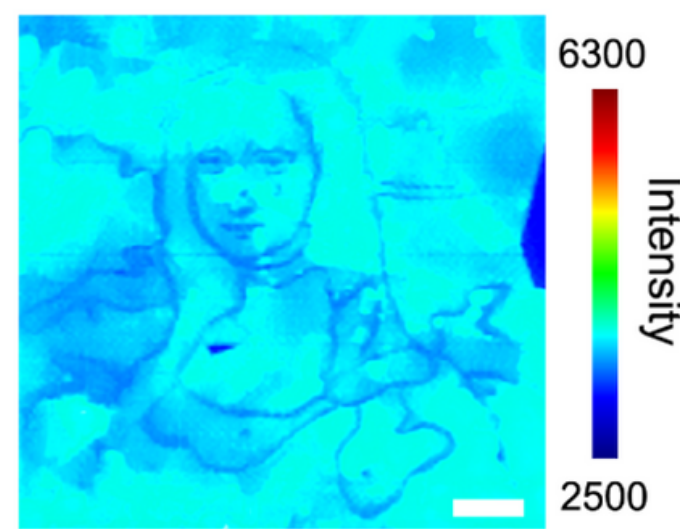

Figure 4

Complex patterns written on continuous film of MoS2 monolayer. a, Optical image of one MoS2 continuous film monolayer. b, A figure of the phrase "Nothing is Impossible" used as a pattern. c, The PL image corresponding to a that has been written with the phrase by laser. $d$, Optical image of another monolayered MoS2 continuous film. The red rectangle denotes the area being mapped. e, A sketch of the famous painting Mona Lisa used as a pattern. $f$, The PL image corresponding to $d$ showing the Mona Lisa painting created by laser writing. Scale bars are all $10 \mu \mathrm{m}$.

\section{Supplementary Files}

This is a list of supplementary files associated with this preprint. Click to download.

- SI20201118.docx 